\title{
Hacia una Ontología para el Gobierno de Desarrollo de Software en Pymes
}

\author{
Toward an Ontology for Software Development \\ GovernANCE IN SMES
}

\author{
${ }^{1}$ Jhon Masso, ${ }^{2}$ César Pardo \\ Facultad de Ingeniería Electrónica y Telecomunicaciones, \\ Universidad del Cauca, Popayán, Colombia \\ ${ }^{1}$ masso@unicauca.edu.co \\ ${ }^{2}$ cpardo@unicauca.edu.co
}

Recibido: 30/05/2014 - Aprobado: 22/06/2014

\section{ResUMEN}

Actualmente, las organizaciones con el fin de mejorar su competitividad y hacerla sostenible en el tiempo, utilizan modelos y estándares que les permiten mejorar sus procesos y la calidad de sus productos. Algunos de estos modelos dirigen su atención hacia el gobierno de Tecnologías de la Información. Sin embargo, y aunque estos describen buenas prácticas de gobierno aplicables a cualquier tipo de organización de $\mathrm{Tl}$, estas no son apropiadas para las exigencias de los proyectos ni para las empresas de desarrollo de software. En este sentido, y con el objetivo de aportar una solución, en este artículo se presenta una primera aproximación de una ontología llamada Onto-GDS la cual ha sido definida por medio de la aplicación del Formalismo para la Representación de Ontologías para Ingeniería de Software (más conocida como Representation Formalism for Software Engineering Ontologies - Refseno). El propósito de Onto-GDS es proporcionar un conjunto de conceptos, relaciones y vocabulario que permitan solucionar las dudas alrededor del gobierno de desarrollo de software.

Palabras clave: gobierno, gobierno de desarrollo de software, GDS, ontología, pymes.

\section{Abstract}

Currently, companies in order to improve their competitiveness and make it sustainable in time, these make use of models and standards, which enable organizations to improve their processes and products development quality. However, although these models describe best practices of IT Governance applicable to any IT organization, these practices are unsuitable for project requirements and software development companies. In this sense and in the order to provide a solution, this paper provides an overview of an ontology named Onto-GDS which has been defined by the application of a Representation Formalism for Software Engineering Ontologies for Software Engineering (better known as REFSENO). The purpose of Onto-GDS is to provide a set of concepts, relationships and vocabulary to resolve the doubts about government software development.

Keywords: governance, software development governance, SDG, ontology, smes. 


\section{InTRODUCCIÓN}

En la actualidad, con el fin de innovar y mejorar sus procesos, las organizaciones utilizan modelos y estándares que ofrecen mejores prácticas para optimizar sus diferentes gestiones, haciéndolas más competitivas en los mercados nacional e internacional. Algunos ejemplos de mejores prácticas bastante difundidas y orientadas a brindar soporte al gobierno corporativo y gestión de las tecnologías de la información (TI) son:

Cobit, un marco de buenas prácticas definido por la Asociación Profesional Internacional de Isaca que permite generar valor a las organizaciones mediante el buen gobierno y gestión de las tecnologías de la información (TI) [1]; ITIL, que provee un conjunto de prácticas para la gestión de servicios de $\mathrm{Tl}$ enfocadas en alinear dichos servicios con las necesidades de las empresas [2]; ISO 20000-2 [3] proporciona orientación sobre la aplicación de sistemas de gestión de servicios con base en los requisitos de la norma ISO 20000-1 [4]; ISO 27001 es un marco que proporciona un conjunto de mejores prácticas para un sistema de gestión de seguridad de la información (SGSI) [5]; ISO 38500 proporciona un marco para la gobernabilidad efectiva de Tl; por ejemplo, cumplir con obligaciones legales y regulaciones éticas en relación con el uso de las TI de sus organizaciones [6] y AS/NZS 8016 que proporciona principios rectores sobre la gobernabilidad de $\mathrm{TI}$ en proyectos [7].

El gobierno a un nivel corporativo tiene como propósito decretar las directrices para llevar a cabo la dirección, administración y control de una corporación, con el fin de satisfacer a los principales stakeholders y entes reguladores [8]. El gobierno corporativo es el encargado de alcanzar los objetivos y mantener el patrimonio de la organización; así, como también, de velar por los intereses de cada uno de los stakeholders a quienes deberá ofrecer transparencia en la ejecución de cada una de las prácticas administrativas y control de la entidad. Es decir, el gobierno corporativo es el sistema por el cual las organizaciones son dirigidas y controladas, haciendo énfasis sobre el uso de componentes como liderazgo y estructuras y procesos organizacionales que permiten que estas se sostengan, y extiendan sus estrategias y objetivos hacia un mejor gobierno organizacional [9].

La Asociación de Control y Auditoría de Sistemas de Información (más conocida por sus siglas en inglés como Isaca) describe el gobierno corporativo como un conjunto de responsabilidades y prácticas ejecutadas por un consejo directivo y la administración, que tiene como fin: proveer dirección estratégica; el aseguramiento del alcance de los objetivos de la organización; la gestión de riesgos de manera adecuada y la verificación de que los recursos de la organización sean utilizados de forma responsable [1]. Por otro lado, la gestión dentro del gobierno de TI es la encargada de la operación del día a día y de los proyectos que se estén llevando a cabo en la empresa [10]. En este sentido, la gestión tiene como objetivo controlar, supervisar y tomar decisiones respecto de las directrices que fijen el cuerpo de gobierno de la organización, el cual estará conformado por el director, propietarios, miembros de la junta, socios, ejecutivos senior o similares, además de los funcionarios autorizados para llevar a cabo las tareas de legislación o regulación. En otras palabras, la gestión tiene la obligación de controlar una organización o parte de ella; así mismo, estará sometida a las políticas y monitorización establecidas por el gobierno corporativo [6].

Es importante mencionar que este conjunto de iniciativas han sido orientadas a empresas de base tecnológica con el fin de brindar soporte a la definición de políticas y procedimientos, así como a la definición y asignación de roles y 
responsabilidades en la utilización de las $\mathrm{TI}$ a dichas prácticas, garantizando la calidad y competencia en su uso. La incorporación de estos mecanismos al desarrollo de software originan el concepto de Gobierno de Desarrollo de Software (GDS), que, una vez implementado, permitirá: mejorar la capacidad para hacer frente a los riesgos; brindar soporte a la toma de decisiones; asegurar que los resultados estén acordes con los objetivos y estrategias de la organización; que el gobierno de software, elemento clave y esencial, permita guiar los proyectos de las organizaciones dedicadas al desarrollo de software, y producir valor agregado a través de la entrega temprana y continua de resultados con base en las especificaciones de los clientes y en las necesidades del negocio [11],[12], [13]; que los proyectos generen valor agregado y, por último, que las organizaciones mejoren continuamente mediante la definición y el establecimiento de estrategias para el seguimiento y control de sus procesos a fin de llevar a cabo una medición y evaluación del desempeño en la realización de los mismos.

Teniendo en cuenta lo anterior, mediante este artículo se presenta una propuesta de una ontología basada en UML como lenguaje de modelado de sistemas de software que permita a los profesionales y miembros de las pequeñas y medianas empresas (PyMEs) su utilización para emprender actividades de implementación y despliegue de prácticas de GDS. Se ha usado UML dado que es un lenguaje que los profesionales e investigadores de la industria de software comprenden fácilmente.

Además de la presente introducción, este artículo se compone de las siguientes secciones: en la Sección II se da a conocer el método de investigación utilizado para este estudio; la Sección III presenta los trabajos relacionados; la Sección IV, la caracterización del conocimiento sobre el GDS mediante una ontología de GDS en PyMEs y, finalmente, en la Sección V se presentan algunas conclusiones y trabajos futuros. Con los resultados obtenidos, se espera proporcionar un vocabulario que solucione las dudas y diferencias entre las distintas definiciones de los conceptos relacionados con el gobierno de desarrollo de software.

\section{MÉtodo de Investigación}

El enfoque metodológico utilizado para llevar a cabo la realización de esta propuesta es Investigación-Acción. Este enfoque permite la adquisición de nuevo conocimiento basado en la prueba de hipótesis, haciendo uso de la interpretación, estudio de datos cualitativos y una serie de ciclos incrementales que permiten obtener los productos de un proyecto de investigación. Este método de investigación fue introducido en el campo de la ingeniería por Wood-Harper para llevar a cabo investigaciones en sistemas de información e ingeniería del software [14][15]. Para la definición de la ontología se aplicó el Formalismo para la representación de ontologías para Ingeniería de Software (Refseno) [16]. En la sección IV se describe de manera más detallada la forma como se diseñó Onto-GDS a partir del formalismo utilizado.

\section{Trabajos Relacionados}

Con base en un análisis preliminar de la literatura, ha sido posible encontrar un notable interés en el incremento de trabajos relacionados y orientados a la definición de propuestas para soportar el gobierno del desarrollo de software.

En la Tabla 1 se presentan las características principales de algunos de los trabajos encontrados: 
TABLA I

Principales Características de Trabajos Relacionados

\begin{abstract}
No. Autores
Principales Características

En este trabajo se describe el GDS como un conjunto de mecanismos que permitan asistir a la organización en la especificación y el establecimiento de las decisiones de gobierno, la determinación

1 [17] de políticas y medidas para controlar y gestionar el comportamiento dinámico de la organización y la evaluación como un elemento primordial para redefinir las metas a través de proyectos de mejora continua.

Propone una visión general del GDS en la que centran sus esfuerzos en dejar en claro los términos de gobierno, gestión y procesos, así como también la estrecha relación que estos mecanismos tienen. Los procesos de gobierno son dirigidos por la estrategia que ha sido definida para una empresa. A su vez, el gobierno es el encargado de establecer los derechos de decisión y las políticas sobre las

$2 \quad[18]$ estructuras de gestión, con el fin de asignar las responsabilidades y autoridad para la toma de decisiones y los mecanismos de medida que permitirán medir los resultados y verificar que estos estén alineados con la estrategia y objetivos de la empresa. Por otro lado, están las estructuras de gestión que serán las encargadas de desplegar los procesos con el propósito de implementar las políticas de gobierno y trabajar hacia la consecución de las metas estratégicas de la empresa.
\end{abstract}

Señala que el gobierno es la clave para obtener el control sobre los procesos del negocio y de los activos resultantes de la ejecución de los mismos. Sus principios son alinear estratégicamente todos

3 Ericsson los recursos con las estrategias del negocio y gestionar los riesgos en las operaciones e inversiones [19] de la organización. Además, da a conocer la perspectiva de gobierno desde el punto de vista del gobierno empresarial, gobierno de TI, gobierno del desarrollo del producto y gobierno del desarroIlo. Mecanismos que ofrecen un conjunto de buenas prácticas y herramientas para llevar a cabo el gobierno de los procesos del negocio, de entregas de sistemas y software.

Miembros del IBM Software Group presentan una iniciativa la cual consiste en aplicar los principios ágiles y la filosofía Lean al gobierno del desarrollo de software en organizaciones TI con el fin de generar valor y rapidez y mejorar la ejecución de las actividades de desarrollo. En esta iniciativa Ambler se detallan 18 prácticas específicas para realizar el gobierno del desarrollo software Lean alineadas

4 y Kroll

[20] con 6 áreas claves del gobierno TI. Además, describen que las principales ventajas que brinda esta práctica a las organizaciones son: promover la difusión efectiva del gobierno con el objetivo de que los equipos TI cumplan con el programa de gobierno de la organización. Hacer factible el gobierno en la organización. Que los procesos y políticas cobren vida para ayudar a la organización a cumplir con sus objetivos teniendo en cuenta el entorno y las personas involucradas [20].

Presenta un framework conceptual soportado en una herramienta tecnológica que posibilita el vínculo entre el desarrollo de software ágil, los negocios a largo plazo y la planificación del producto y las entregas, elementos que brindarían soporte al GDS. El framework propuesto permite a los usuaJarno rios la identificación de los roles necesarios, las responsabilidades y las estructuras para la toma de Vähä- $\quad$ decisiones en la alta gerencia del negocio y la priorización del trabajo diario de los desarrolladores. niitty La herramienta que soporta esta iniciativa fue denominada, Agilefant, la cual facilita la gestión de [19] proyectos software de extremo a extremo (end-to-end) mediante una propuesta ágil como Scrum que ofrece un marco de trabajo iterativo e incremental para el desarrollo de proyectos, productos y aplicaciones, junto con el framework de escalado ágil (más conocido por sus siglas en inglés como $\mathrm{SAFe}$ ) que permite un marco de trabajo para aplicar principios Lean y Agile en el ámbito empresarial.

Presentan una propuesta en la que se exhiben una metodología y un conjunto de buenas prácticas basadas en las normas ISO para ejercer el gobierno y la gestión de las tecnologías y los sistemas de información (TSI), cuyo objetivo es brindar a los responsables de las organizaciones elementos

AENOR necesarios para llevar a cabo un buen gobierno mediante la optimización de los recursos, la articu-

[10] lación eficiente del modelo de gobierno y de gestión de las TIC, incorporando la calidad y seguridad en los servicios y proyectos, además de prácticas para llevar a cabo la evaluación, mejora y madurez del software. Estos elementos permiten cumplir con los objetivos definidos en el plan estratégico de la organización. 
A partir del análisis de los trabajos relacionados, se puede observar que el gobierno de desarrollo de software (GDS) es un nuevo campo de investigación que ha estado a la sombra del concepto de gobierno y gestión de Tl; sin embargo, estos tienen grandes diferencias. El gobierno de TI brinda soporte a las necesidades y objetivos del negocio mediante la definición de políticas y procedimientos para la asignación de roles y responsabilidades en la utilización de las TI, garantizando la calidad y competencia en la utilización de las mismas. Por ejemplo, modelos como Cobit se centran más en controlar y optimizar la inversión en TI que en la ejecución de procesos de desarrollo de software, con el fin de que empresas de TI soporten los objetivos de su negocio sobre procesos concretos y apropiados. Por su parte, en ITIL se proporciona un conjunto de prácticas para la gestión de servicios de TI basada en la infraestructura tecnológica que poseen las organizaciones. La ISO 38000 provee un conjunto de procesos para llevar a cabo el gobierno corporativo de $\mathrm{TI}$ con el ánimo de dirigir y controlar el uso actual y futuro de las TI. Igualmente, un conjunto de procesos y controles para llevar a cabo la gestión de TI y cumplir con los objetivos de la organización. Adicionalmente, la ISO 20000-2 ofrece las mejores prácticas para la gestión de servicios de TI y aplicación de sistemas de gestión de servicios [3].

Por su parte, el gobierno de desarrollo de software brinda los mecanismos claves para la toma de decisiones y la satisfacción de las políticas de gerencia enfocadas en el producto y la generación de valor por el cumplimiento de las estrategias del negocio mediante la gestión y realización de cada uno de los proyectos de desarrollo de software. Prácticas que permiten incrementar la eficiencia en la ejecución de cada una de las actividades de la organización, además de hacerlas sostenibles en el tiempo, todo esto desde un enfoque de organización orientada al desarrollo de software.

Aunque el software como producto hace parte de las TI, y las actividades de desarrollo de software han empezado a incluirse en las iniciativas de gobierno de TI, el GDS [18] debe preocuparse por: administrar el valor: alineando el negocio y el software a nivel organizacional o a través de los diferentes niveles de los proyectos, equilibrar los riesgos y las ganancias, proveer la claridad y la rendición de cuentas; el desarrollo flexible: mediante el apalancamiento de los recursos globales para permitir la elección del desarrollo ágil y el uso de procesos iterativos para reducir el riesgo y el control de riesgos y cambios: midiendo continuamente para reducir los riegos, permitir la gestión del cambio en el ciclo de vida y conocer las necesidades de cumplimiento internas y externas. Mecanismos que sin duda permitirán un buen gobierno y gestión integral de una organización dedicada al desarrollo de software.

Por último, las iniciativas orientadas al GDS proponen una descripción conceptual y estructural a muy alto nivel que, si bien, no se centra de lleno al GDS, tiene como objetivo el gobierno de TI. En este sentido, y con el objetivo de aportar una solución que facilite el GDS en las PyMEs, se propone la definición de la ontología Onto-GDS, que permite identificar y caracterizar el conocimiento existente sobre el gobierno de desarrollo de software. Además, los profesionales y PyMEs podrán hacer uso de ella para las actividades de implementación y el despliegue de mejores prácticas como soporte al gobierno de desarrollo de software. Esto permitirá impactar sobre el incremento de la productividad y competitividad en este tipo de organizaciones.

\section{Onto-Gds: Ontología PARA El Gobierno de Desarkollo de Software}

A continuación se presenta una aproximación general de una ontología para el gobierno de desarrollo de software en PyMEs en términos de su propósito y visión general de los componentes básicos que la conforman. 


\section{A.Método utilizado para la definición de Onto-GDS}

Existe un conjunto amplio de metodologías para facilitar la definición de ontologías. Por ejemplo: la ontología basada en la gestión del conocimiento Methontology [21], [22]; el enfoque para la definición de ontologías portables [23] y la metodología para la definición de ontologías de primer orden lógico [24], entre otras. Teniendo en cuenta lo anterior y después de estudiar las diferentes metodologías para la definición de ontologías, se ha decidido utilizar para la de Onto-GDS el Formalismo para la representación de ontologías para Ingeniería de Software (Representation Formalism for Software Engineering Ontologies - Refseno) [16], dado que es una ampliación y adaptación de Methontology, la cual se usa bastante para la definición de ontologías con diferentes campos de aplicación. Además, ha sido particularmente diseñada como una especialización de Methontology para la creación de ontologías en la ingeniería de software por medio de constructores que permiten describir conceptos, atributos y relaciones entre ellos (Refseno utiliza tres tablas especialmente diseñadas para representar estos elementos: glosario de conceptos, atributos y relaciones), a diferencia de otras metodologías y enfoques que describen otras representaciones menos intuitivas para la definición de ontologías con predicados lógicos de primer orden.

Refseno es mucho más intuitiva dado que hace una distinción entre los niveles de conocimiento en cuanto a lo contextual y el contexto específico y, porque, además, provee diferentes técnicas para el análisis de la consistencia de la ontología e instancias en relación con la implementación.

\section{B. Propósito}

El propósito de esta ontología es la identificación y unificación del conocimiento existente en la literatura sobre el gobierno de desarrollo de software. La ontología involucra los principales conceptos que podrán ser utilizados por las PyMES de software, para definir las mejores prácticas e implementar y desplegar procesos de gobierno de desarrollo de software, con el fin de lograr el cumplimiento de sus objetivos estratégicos. De la misma manera, ofrece un conjunto de elementos que deberán ser tenidos en cuenta para que este tipo de iniciativas sean formalizadas y sostenibles en el tiempo y así mejorar la capacidad de la organización en el uso efectivo de sus recursos.

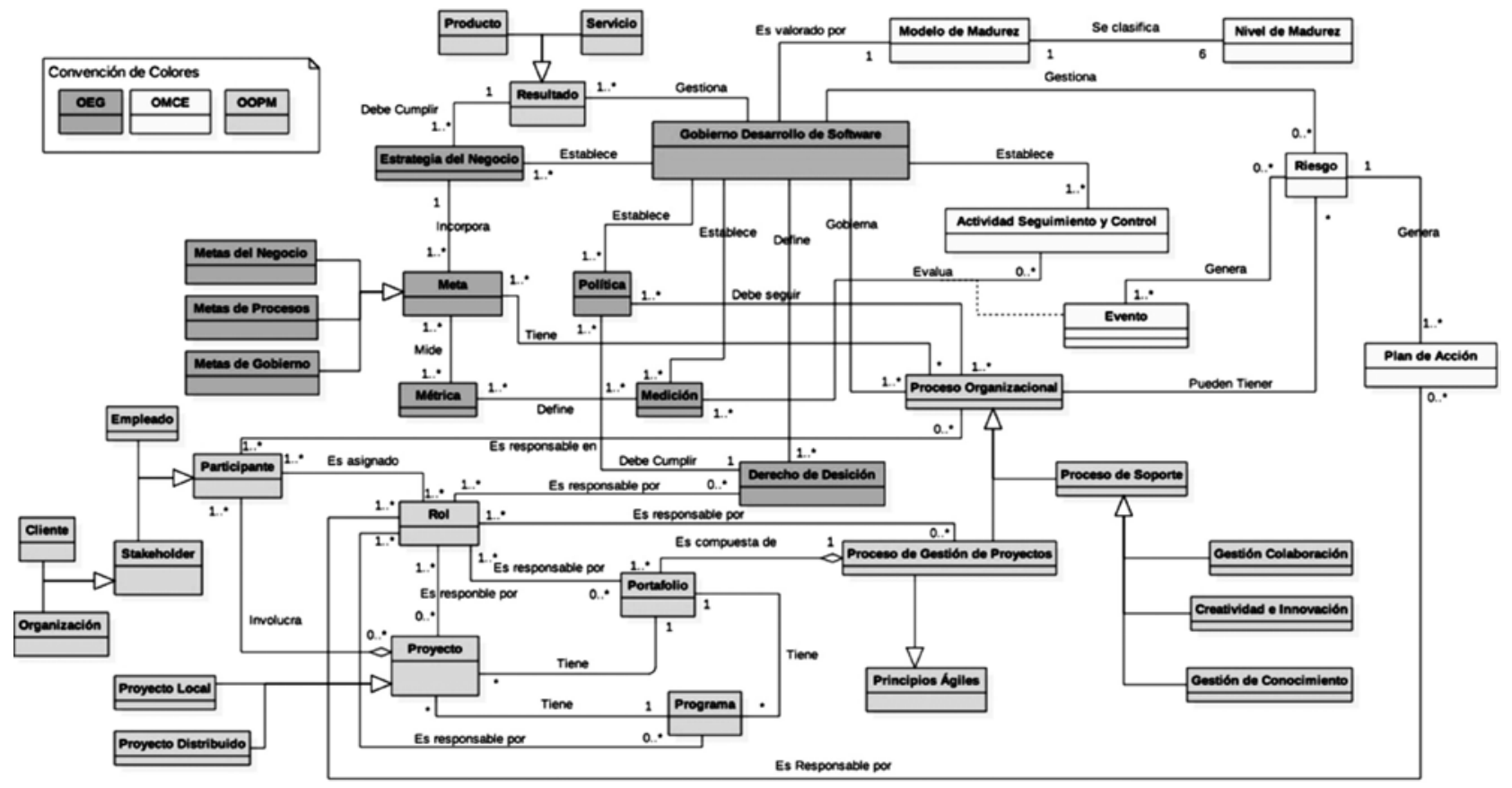

Fig. 1 Ontología para el Gobierno de Desarrollo de Software (Onto-GDS). 


\section{Visión general de la Ontología}

En la Fig. 1 se muestra la ontología construida, la cual está conformada por tres sub-ontologías: Ontología para el establecimiento de gobierno (Ontology for Establishment of Governance - OEG), la cual brinda soporte a la especificación y el establecimiento de las estructuras de gobierno; Ontología para el seguimiento, control y evaluación (Ontology for monitoring, control and evaluation - OMCE), que brinda soporte al seguimiento, control y evaluación del gobierno de desarrollo de software y Ontología para la gestión de procesos organizacionales (Ontology for Organizacional Process Management - OOPM), que soporta la gestión de los procesos organizacionales involucrados en el GDS. En la Fig. 1 se puede apreciar las principales relaciones entre cada uno de los conceptos identificados en el GDS y una descripción de cada una de las sub-ontologías con el fin de dar a conocer su propósito general. Dado las limitaciones de espacio, una versión más clara de la ontología se puede ver a través del siguiente link: https://goo.gl/nmbkFl. A continuación se describe cada una de ellas:

\section{1) Ontology for Establishment of Governan-} ce (OEG): en esta sub-ontología se encuentran los conceptos relacionados con el establecimiento de la estrategia de negocio en función de los intereses de la organización y del GDS; los mecanismos que permitirán la definición y establecimiento de las metas y políticas de la organización respecto de los diferentes procesos organizacionales y los conceptos orientados a la instauración de los mecanismos de medición y la declaración de los diferentes roles y responsabilidades para llevar a cabo la toma de decisiones. La Fig. 2 muestra en mejor detalle las relaciones y conceptos de la ontología OEG.

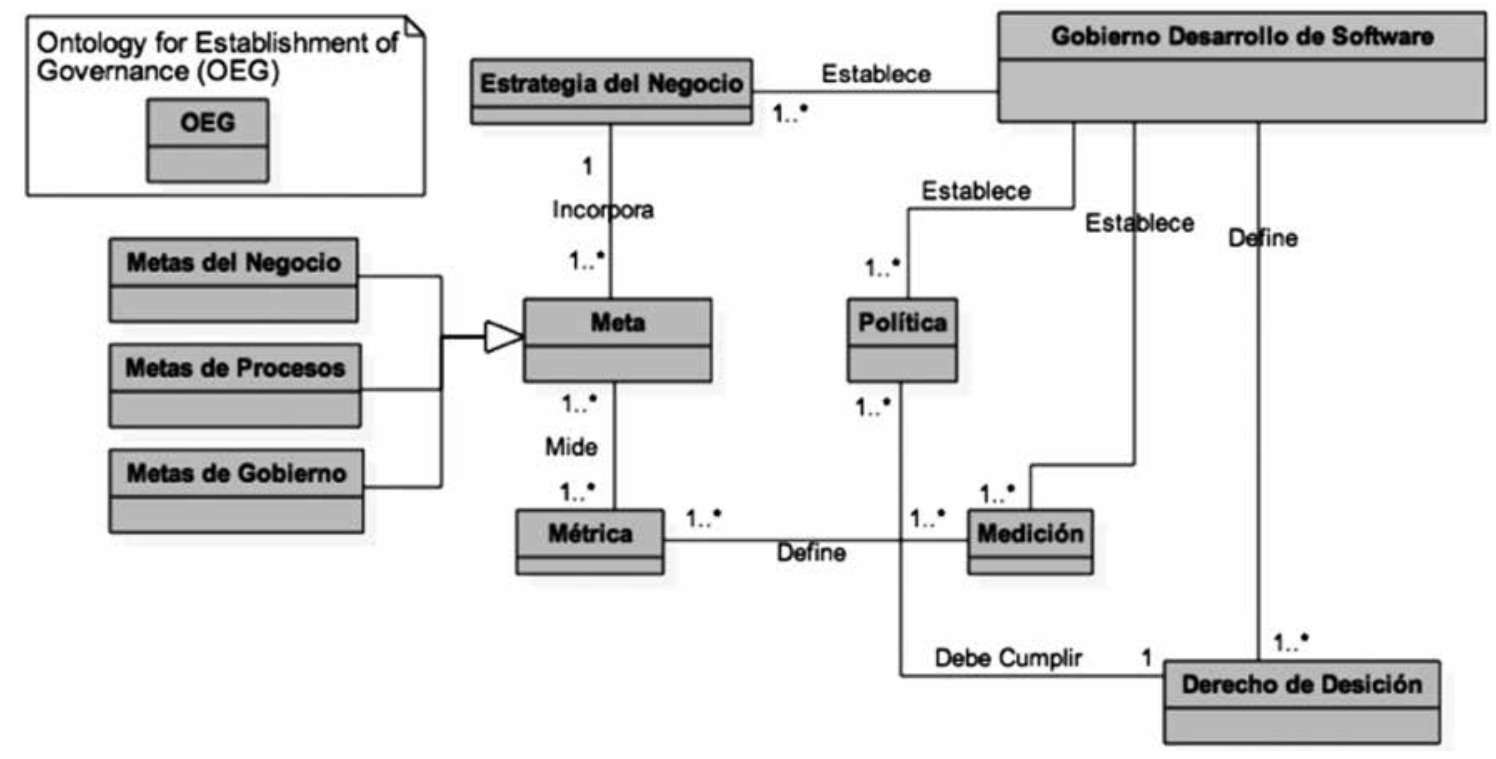

Fig. 2 Ontología para el Establecimiento de Gobierno (OEG).

2) Ontology for monitoring, control and evaIuation (OMCE): en esta sub-ontología han sido modelados los principales conceptos que permitirán llevar a cabo la vigilancia del cumplimiento de todas las metas del negocio mediante las actividades de seguimiento y control. Lo anterior, con el objetivo de tener en cuenta los conceptos que permitirán evaluar las capacidades y el cumplimiento en función de la medición del desempeño, y la identificación de las oportunidades de mejora. Así mismo, se incluyen los conceptos destinados a la gestión y control de riesgos. La Fig. 3 muestra en mejor detalle las relaciones y conceptos de la ontología OMCE. 
3) Ontology for Organizacional Process Management (OOPM): en esta sub-ontología se han identificado los principales conceptos relacionados con los procesos organizacionales que serán gobernados y gestionados por el GDS. En los conceptos identificados se encuentran los procesos de soporte orientados hacia la búsqueda de nuevas oportunidades de innovación en los productos, servicios y prácticas llevadas a cabo en la organización. También, se identifican los conceptos relacionados con la gestión de técnicas de creatividad, colaboración e innovación, junto a la transferencia y apropiación del conocimiento mediante la gestión de cada unos de los activos de la organización. La Fig. 4 muestra en mejor detalle las relaciones y conceptos de la ontología OOPM.

Por otro lado, se han identificado los conceptos relacionados con el proceso de gestión de proyectos que brindarán soporte al enfoque de GDS y permitirán obtener resultados que deberán estar alineados a las estrategias del negocio. Este proceso apropiará los principios ágiles para la gestión de proyectos y de desarrollo de software.

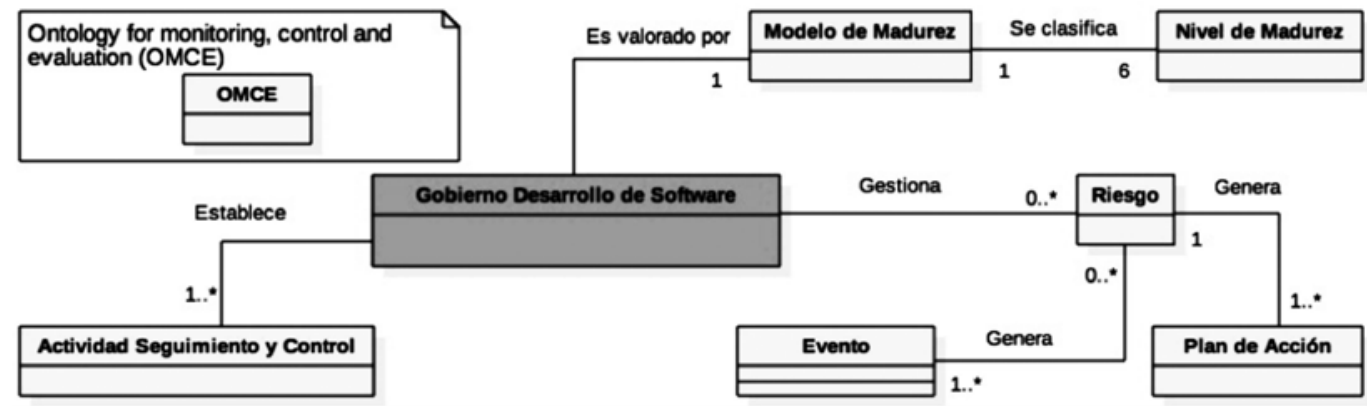

Fig. 3 Ontología para el monitoreo, control y evaluación de Gobierno (OMCE).

\section{Definición de los términos in Onto-GDS}

La Tabla 2 presenta los términos, definición y fuente de algunos términos identificados en cada una de las sub-ontologías que se proponen en Onto-GDS. Los términos han sido identificados a partir de la lectura y el análisis de los trabajos relacionados. Los términos y definiciones son propuestas referenciadas en su totalidad de otros autores o adaptaciones de ellas. Asimismo, algunas definiciones son propias.

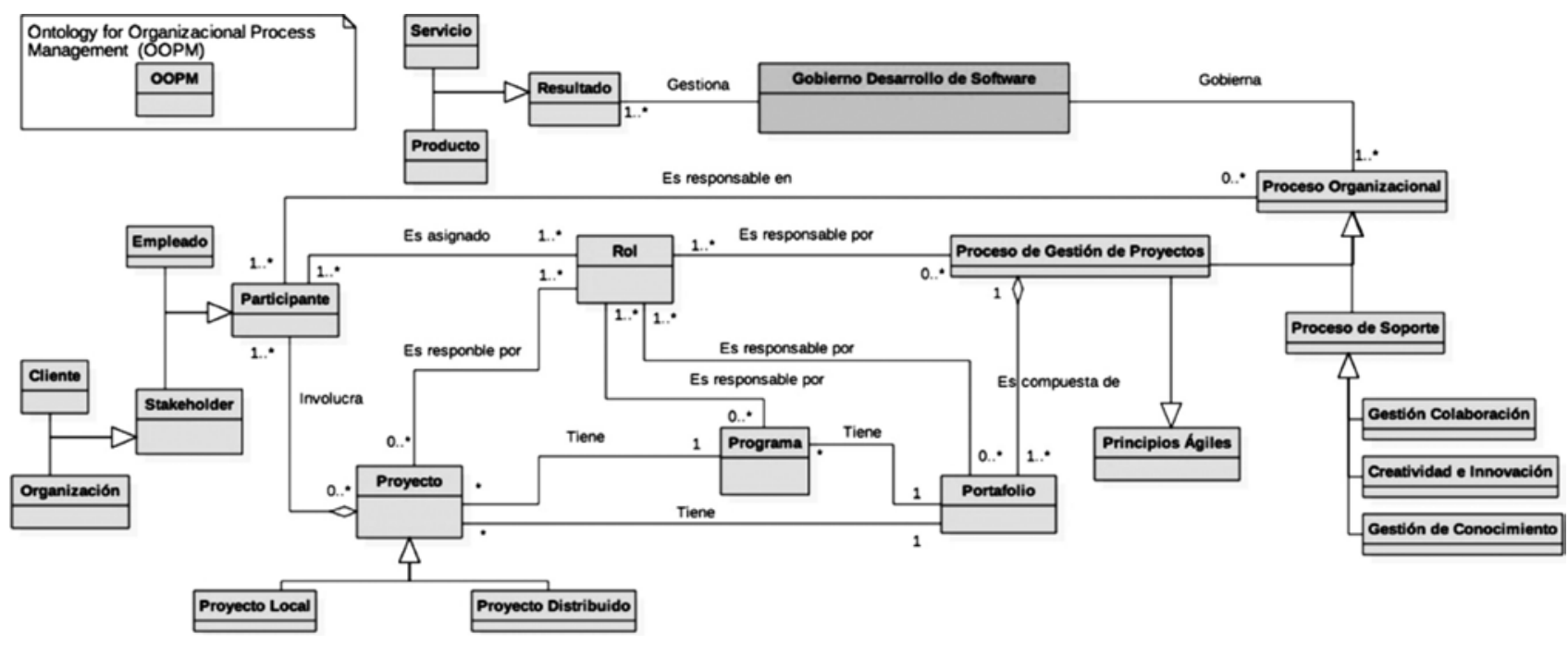

Fig. 4 Ontología para la Gestión de los Procesos Organizacionales (OOPM). 
TABLA II

Definición de los Términos Onto-GDS

\begin{tabular}{|c|c|c|c|}
\hline Término & $\begin{array}{c}\text { Súper } \\
\text { concepto }\end{array}$ & Definición & Fuente \\
\hline $\begin{array}{l}\text { Gobierno de } \\
\text { desarrollo de } \\
\text { software }\end{array}$ & Concepto & $\begin{array}{l}\text { Conjunto de mecanismos que permiten asistir a la organización } \\
\text { en la especificación y establecimiento de las decisiones de go- } \\
\text { bierno relacionadas con la determinación de políticas y medidas } \\
\text { para controlar y gestionar el comportamiento dinámico de la } \\
\text { organización, y con la evaluación como un elememto primordial } \\
\text { para redefinir las metas a través de proyectos de mejora con- } \\
\text { tinua. }\end{array}$ & $\begin{array}{l}\text { Adaptado } \\
\text { de: [18] }\end{array}$ \\
\hline \multicolumn{4}{|c|}{ OEG } \\
\hline Métrica & Concepto & $\begin{array}{l}\text { Conjunto de medidas destinadas a ayudar a gestionar los } \\
\text { procesos involucrados en el gobierno de desarrollo de software } \\
\text { que permiten medir el desempeño respecto de todas las metas. }\end{array}$ & $\begin{array}{l}\text { Adaptado } \\
\text { de: [9] }\end{array}$ \\
\hline Medición & Concepto & $\begin{array}{l}\text { Es un proceso para establecer, planear, ejecutar y evaluar me- } \\
\text { diciones de software dentro de un proyecto o de una estructura } \\
\text { de mediciones en una organización. }\end{array}$ & $\begin{array}{l}\text { Tomado de: } \\
\text { [25] }\end{array}$ \\
\hline Meta & Concepto & $\begin{array}{l}\text { Es un propósito claro de la organización en términos de las } \\
\text { necesidades del negocio, de sus procesos y de las actividades } \\
\text { del gobierno de desarrollo de software. Las metas deben ser } \\
\text { alcanzables y medibles; además, deben ser coherentes con los } \\
\text { recursos que dispone a organización con el propósito de lograr } \\
\text { los objetivos del negocio. }\end{array}$ & $\begin{array}{l}\text { Fuente } \\
\text { propia }\end{array}$ \\
\hline Política & Concepto & $\begin{array}{l}\text { Describe los principios del gobierno de desarrollo de software } \\
\text { a muy alto nivel y estrategias por seguir por parte de la orga- } \\
\text { nización, que tienen como propósito influenciar y guiar la toma } \\
\text { de decisiones a fin de que estas estén alineadas con las metas y } \\
\text { estrategias de la organización. }\end{array}$ & $\begin{array}{l}\text { Adaptado } \\
\text { de: [9] }\end{array}$ \\
\hline $\begin{array}{l}\text { Estrategia } \\
\text { de negocio }\end{array}$ & Concepto & $\begin{array}{l}\text { Es un conjunto de acciones definidas por la alta gerencia que } \\
\text { especifica los lineamientos por seguir por parte de la organiza- } \\
\text { cion. Las estrategias del negocio deberán estar orientadas hacia } \\
\text { la definición de los servicios y productos que se desean desa- } \\
\text { rrollar en la organización, el mercado y los clientes que se desea } \\
\text { satisfacer, al igual que los métodos y herramientas por utilizar } \\
\text { para llevar a cabo el desarrollo de proyectos software. }\end{array}$ & $\begin{array}{l}\text { Fuente } \\
\text { propia }\end{array}$ \\
\hline $\begin{array}{l}\text { Derecho } \\
\text { de decisión }\end{array}$ & Concepto & $\begin{array}{l}\text { Especifica los niveles de autoridad para la toma de decisiones en } \\
\text { la organización respecto de todo el conjunto de procesos invo- } \\
\text { lucrados en el gobierno de desarrollo software. Los derechos de } \\
\text { decisión son una fuente de documentación en la que se describe } \\
\text { de forma detallada todo el conjunto de funciones y responsabi- } \\
\text { lidades que han de seguir los agentes involucrados en cada uno } \\
\text { de los procesos en términos de quiénes son sus encargados y } \\
\text { responsables, quiénes son los encargados de brindar soporte, } \\
\text { los agentes que deben ser consultados e informados respecto } \\
\text { de los progresos en los procesos, actividades y resultados. }\end{array}$ & $\begin{array}{l}\text { Adaptado } \\
\text { de: [26], } \\
{[27]}\end{array}$ \\
\hline
\end{tabular}




\begin{tabular}{|l|l|l|l|}
\multicolumn{1}{|c|}{ Término } & $\begin{array}{c}\text { Súper } \\
\text { concepto }\end{array}$ & \multicolumn{1}{|c|}{ Definición } & Fuente \\
\hline $\begin{array}{l}\text { Modelo } \\
\text { de madurez }\end{array}$ & Concepto & $\begin{array}{l}\text { Es un framework conceptual que define los niveles de madurez } \\
\text { del gobierno de desarrollo de software respecto de la capacidad } \\
\text { que tiene una organización para el cumplimento de cada una de } \\
\text { las metas de los procesos involucrados. }\end{array}$ & $\begin{array}{l}\text { Adaptado } \\
\text { de: [28] }\end{array}$ \\
\hline $\begin{array}{l}\text { Nivel de } \\
\text { madurez }\end{array}$ & Concepto & $\begin{array}{l}\text { Nivel de la mejora del conjunto de procesos involucrados en el } \\
\text { gobierno de desarrollo de software que determina el cumpli- } \\
\text { miento de los objetivos de cada uno de los procesos. }\end{array}$ & $\begin{array}{l}\text { Adaptado } \\
\text { de: [28] }\end{array}$ \\
\hline Riesgo & Concepto & $\begin{array}{l}\text { El potencial de que una amenaza específica explote las debili- } \\
\text { dades de un activo o grupo de activos para ocasionar pérdida } \\
\text { y/o daño a los activos. Por lo general, se mide por medio de una } \\
\text { combinación del impacto y la probabilidad de ocurrencia. }\end{array}$ & $\begin{array}{l}\text { Tomado } \\
\text { de: [8] }\end{array}$ \\
\hline $\begin{array}{l}\text { Actividad de } \\
\text { seguimiento } \\
\text { y control }\end{array}$ & Concepto & $\begin{array}{l}\text { Establece el conjunto de acciones que permiten llevar a cabo el } \\
\text { seguimiento y control de cada uno de los procesos involucrados } \\
\text { en el gobierno de desarrollo con el fin de evaluar su ejecución } \\
\text { respecto de los mecanismos de medición para así tomar las ac- } \\
\text { ciones pertinentes que permitan su mejora continua. }\end{array}$ & $\begin{array}{l}\text { Fuente } \\
\text { propia }\end{array}$ \\
\hline Evento & Concepto & $\begin{array}{l}\text { Es una alerta o notificación creada a partir de las actividades } \\
\text { de seguimiento y control que pueden generar riesgos y que re- } \\
\text { quieren que el personal de la organización tome las acciones } \\
\text { pertinentes para solucionarlos. }\end{array}$ & $\begin{array}{l}\text { Adaptado } \\
\text { de: [2] }\end{array}$ \\
\hline $\begin{array}{l}\text { Plan de } \\
\text { acción }\end{array}$ & Concepto & $\begin{array}{l}\text { Son el conjunto de pasos que ha de seguir la organización para } \\
\text { dar solución a los riesgos generados en la ejecución de los pro- } \\
\text { cesos involucrados en el gobierno de desarrollo de software. }\end{array}$ & $\begin{array}{l}\text { Fuente } \\
\text { propia }\end{array}$ \\
\hline
\end{tabular}

\begin{tabular}{|c|c|c|c|}
\hline \multicolumn{4}{|c|}{ 00PM } \\
\hline Resultado & Concepto & $\begin{array}{l}\text { Es una salida de la ejecución de procesos o actividades llevados } \\
\text { a cabo en una organización. Estos resultados pueden ser pro- } \\
\text { ductos cuantificables ya terminados o a nivel de componentes. } \\
\text { En el caso de los servicios será un trabajo útil que no produce } \\
\text { un producto ni un resultado tangible. }\end{array}$ & $\begin{array}{l}\text { Adaptado } \\
\text { de: [29] }\end{array}$ \\
\hline Producto & Resultado & $\begin{array}{l}\text { El conjunto de artefactos por ser desarrollados, entregados y } \\
\text { mantenidos en un proyecto. Los productos pueden ser de salida } \\
\text { o entrada, mandatorios u opcionales. Los productos en la ma- } \\
\text { yoría de los casos de artefactos tangibles utilizados, producidos } \\
0 \text { modificados por áreas. }\end{array}$ & $\begin{array}{l}\text { Tomado } \\
\text { de: }[30]\end{array}$ \\
\hline $\begin{array}{l}\text { Proceso or- } \\
\text { ganizacional }\end{array}$ & Concepto & $\begin{array}{l}\text { Conjunto de procesos que permiten soportar las actividades de } \\
\text { gestión de la organización con el fin de cumplir con las metas y } \\
\text { estrategias. }\end{array}$ & $\begin{array}{l}\text { Fuente } \\
\text { propia }\end{array}$ \\
\hline $\begin{array}{l}\text { Principios } \\
\text { ágiles }\end{array}$ & Concepto & $\begin{array}{l}\text { Conjunto de valores que permiten a las organizaciones dedica- } \\
\text { das al desarrollo de software satisfacer las necesidades de los } \\
\text { clientes mediante la entrega temprana y oportuna de productos } \\
\text { software que generen valor y que han sido desarrollados me- } \\
\text { diante el uso de metodologías ágiles. }\end{array}$ & $\begin{array}{l}\text { Adaptado } \\
\text { de: [31] }\end{array}$ \\
\hline
\end{tabular}




\begin{tabular}{|c|c|c|c|}
\hline Término & $\begin{array}{l}\text { Súper } \\
\text { concepto }\end{array}$ & Definición & Fuente \\
\hline $\begin{array}{l}\text { Proceso } \\
\text { de gestión } \\
\text { de proyectos }\end{array}$ & Concepto & $\begin{array}{l}\text { Consiste en la aplicación de habilidades, herramientas y técni- } \\
\text { cas a las actividades del proyecto con el fin de cumplir con los } \\
\text { requisitos del mismo. La gestión de proyectos se logra median- } \\
\text { te la aplicación e integración de los procesos de gestión de pro- } \\
\text { yectos de inicio, planificación, ejecución, seguimiento y control } \\
\text { y cierre. Desde un contexto más amplio, la organización deberá } \\
\text { incluir la gestión de programas y portafolios para lograr el cum- } \\
\text { plimiento de sus metas mediante la gestión y ejecución de cada } \\
\text { uno de los proyectos de desarrollo de software. }\end{array}$ & $\begin{array}{l}\text { Adaptado } \\
\text { de: [29] }\end{array}$ \\
\hline Proyecto & Concepto & $\begin{array}{l}\text { Es un esfuerzo temporal que se lleva a cabo para crear un pro- } \\
\text { ducto, servicio o resultado único con base en un cronograma } \\
\text { y presupuesto acordados. En el contexto de una organización } \\
\text { dedicada al desarrollo software, los proyectos de desarrollo se } \\
\text { pueden realizar de forma local o distribuida siguiendo el para- } \\
\text { digma del desarrollo de software global. }\end{array}$ & $\begin{array}{l}\text { Adaptado } \\
\text { de: [8], } \\
{[29],[32]}\end{array}$ \\
\hline Rol & Concepto & $\begin{array}{l}\text { Describe un conjunto o grupo de responsabilidades, deberes o } \\
\text { habilidades requeridas para la ejecución de una actividad espe- } \\
\text { cífica. }\end{array}$ & $\begin{array}{l}\text { Tomado } \\
\text { de: [30] }\end{array}$ \\
\hline Participante & Concepto & $\begin{array}{l}\text { Hace referencia al conjunto de agentes que participan en cual- } \\
\text { quier proceso, actividad y tarea en una organización. Estos } \\
\text { agentes podrán ser empleados y stakeholders. Para el caso de } \\
\text { los stakeholders pueden ser del tipo clientes (personas natu- } \\
\text { rales) y organizaciones (personas jurídicas). }\end{array}$ & $\begin{array}{l}\text { Fuente } \\
\text { propia }\end{array}$ \\
\hline
\end{tabular}

\section{E. Ejemplo de aplicación de la Ontología}

Un ejemplo teórico de aplicación de algunos de los conceptos propuestos por Onto-GDS se puede observar a continuación. Los conceptos utilizados de la ontología se pueden apreciar en letra cursiva: una organización después de tener claro cómo se encuentra y hacia dónde quiere llegar a nivel organizacional y como unidad de negocio, deberá llevar a cabo el establecimiento de sus estrategias del negocio para poder lograr sus expectativas. Estas estrategias deberán estar orientadas hacia la definición de los servicios y productos que se desean desarrollar, el mercado y clientes que se desea satisfacer, los métodos y herramientas por utilizar en el desarrollo de los proyectos software, entre otros. La organización deberá determinar cuáles serán las metas de cada una de sus estrategias, al igual que el conjunto de métricas que le permitirán medir y evaluar su desempeño con respecto al cumplimiento de las mismas. Este es un proceso dinámico y a la vez complejo pero que una vez institucionalizado deberá permitir a las organizaciones mejorar continuamente. Por otro lado, los proyectos deberán ser gobernados y gestionados con regularidad con el fin de que sus resultados generen valor y estén alineados con las estrategias de la organización. Finalmente, el GDS deberá velar por que los riesgos que se originan de las actividades de gobierno y gestión de los procesos, sean administrados de forma adecuada para, de esta manera, generar un claro entendimiento de los mismos y de los planes de acción pertinentes para su mitigación.

\section{Conclusiones Y TRABAJO FUTURO}

En este trabajo se ha presentado una visión general de Onto-GDS, una ontología para llevar a cabo el Gobierno de Desarrollo de Software en PyMEs. Onto-GDS permite unificar y caracterizar 
el conocimiento existente sobre este campo de la ingeniería de software en el cual se evidencia un creciente interés en la definición de propuestas que permitan facilitar el GDS. La ontología fue definida mediante un modelo UML que permitirá su análisis y adopción por parte de la comunidad científica y de profesionales de las PyMEs. Además, servirá como insumo inicial para llevar a cabo su implementación en un lenguaje formal con el fin de validar las diferentes relaciones entre los conceptos identificados. Onto-GDS, al ser una conceptualización específica como el gobierno de TI, será fundamental para la construcción de marcos, modelos, metodologías o procesos.

Como trabajo futuro, se espera llevar a cabo la validación de la ontología a través de su aplicación mediante la creación de instancias de objetos a través de un caso teórico y el uso de una herramienta de diseño y modelado de ontologías. Por ejemplo [33], y el diseño de elementos a partir de la ontología que permita definir los procesos para el GDS. Así mismo, se espera llevar a cabo dos estudios de caso aplicados a organizaciones de software que involucren la utilización de la ontología para la definición de la estructura de procesos en sus procedimientos. Además, se espera elaborar la definición de un framework metodológico que facilite la implementación y despliegue de soluciones relacionadas al GDS en PyMEs. Por ejemplo, elementos de proceso relacionados con el conjunto de actividades, tareas, roles y responsabilidades involucradas en el gobierno de desarrollo de software.

Finalmente, el gobierno de desarrollo de software permitirá beneficiar a las empresas de la región y del país en temas relacionados con la mejora de sus procesos desde un enfoque de gobierno que hasta el momento no ha sido abordado. Esta iniciativa se traduce a un siguiente paso de las organizaciones de software en sus proyectos de mejora, otorgándoles grandes beneficios como los mencionados anteriormente en este artículo.

\section{AgradeCIMIENTOS}

Los profesores Jhon Masso y César Pardo agradecen la contribución de la Universidad del Cauca, donde trabajan como profesor auxiliar y profesor asistente, respectivamente.

\section{RefERENCIAS}

[1] ISACA, "COBIT 5: A Business Framework for the Governance and Management of Enterprise IT," 2012.

[2] ITIL, "Information Technology Infrastructure Library V3," 2010. [Online]. Available: http://www.itil-officialsite.com/.

[3] ISO, "ISO 20000-2:2012. Information technology -- Security techniques -- Information security management systems -- Requirements. Technical report," 2012.

[4] ISO, "ISO/IEC 20000-1:2011 Information technology -- Service management -- Part 1: Service management system requirements," 2011.

[5] ISO, "ISO/IEC 27001:2013. Information Security Management System (ISMS) requirements. Technical report," 2013.

[6] ISO, "ISO/IEC 38500:2015 Information technology Governance of IT for the organization Technologies de l'information - Gouvernance des technologies de l'information pour l'entreprise (Second Edition 201502-15 ed.). Technical report," 2015.

[7] AS/NZS, "AS/NZS 8016:2013 Governance of IT enabled projects. Second edition. Technical report," 2013.

[8] P. L. Bannerman, "Software development governance: A meta-management perspective," Softw. Dev. Governance, 2009. SDG '09. ICSE Work., no. IEEE, pp. 3-8, 2009.

[9] ISACA, "Glossary of Terms English - Spanish," 2015. .

[10] C. M. Sánchez Fernández and M. Piattini Velthuis, Modelo para el gobierno de las TIC basado en las normas ISO, First Edit. Madrid: AENOR, 2012.

[11] A. Kofman, A. Yaeli, T. Klinger, and P. Tarr, "Roles, Rights, and Responsibilities: Better Governance Through Decision Rights Automation," in Software Development Governance, 2009. SDG '09. ICSE Workshop on, 2009, pp. 9-14.

[12] Y. Dubinsky, A. Yaeli, E. Zarpas, Y. Feldman, and G. Nechushtai, "Governance of Software Development: The Transition to Agile Scenario," in book chapter in Information Technology Governance and Service Management: Frameworks and Adaptations, 2007.

[13] M. C. and J. D. Sanders, "Operational IT Governance," IBM developerWorks, May-2007. 
[14] T. Wood-Harper, "Research Methods in Information Systems: Using Action Research.", in Research Methods in Information Systems, 1985, pp. 169-191.

[15] N. Kock, Information Systems Action Research An Applied View Of Emerging Concepts And Methods. Laredo, Texas, USA: Springer Science+Business Media, LLC, 2007.

[16] C. Tautz and C. G. Wangenheim, "REFSENO: A Representation Formalism for Software Engineering Ontologies, IESE-Report No. 015.98/E Version 1.1," Kaiserslautern - Germany, 1998.

[17] Y. Dubinsky and P. Kruchten, "Software development governance (SDG): report on 2nd workshop," ACM SIGSOFT Softw. Eng. Notes, vol. 34, no. 5, pp. 46-47, 2009.

[18] S. Chulani, C. Williams, and A. Yaeli, "Software development governance and its concerns," in Proceedings of the 1st international workshop on Software development governance (SDG '08), 2008, pp. 3-6.

[19] M. Ericsson, "The governance landscape: Steering and measuring development organizations to align with business strategy," 2007.

[20] S. W. Ambler and Per Kroll, "Applying agile and lean principles to the governance of software and systems development White paper- Lean development governance," 2007. [Online]. Available: http://goo.gl/2IZtgm.

[21] D. Fensel, "Ontology-based knowledge management," Computer, vol. 35, no. 11, pp. 56-59, 2002.

[22] M. Fernández, A. Gómez-Pérez, and N. Juristo, "METHONTOLOGY: from ontological art towards ontological engineering," in Proceedings of the AAA197 Spring Symposium Series on Ontological Engineering, 1997, pp. 33-40.

[23] T. R. Gruber, "A translation approach to portable ontology specifications," Knowl. Acquis. - Spec. issue Curr. issues Knowl. Model., vol. 5, no. 2, pp. 199220, 1993.
[24] T. Hikita and M. J. Matsumoto, "Business process modelling based on the ontology and first-order logic," in Proc. 3rd Int. Conf. on Enterprise Information Systems (ICEIS'2001), 2001, pp. 717-723.

[25] ISO, "ISO/IEC 15939:2007 - Information technology - Software engineering - Software measurement process," 2007.

[26] PMI, A Guide to the Project Management Body of Knowledge ( $P M B O K^{\circledR}$ Guide ), Third Edit. Newton Square, Pennsylvania USA: Project Management Institute Inc, 2004.

[27] A. Petrakova and P. Reusch, "Analysis of project stakeholders with the help of RASCI Matrix (on the example of Project Management processes applied at Business Division Electronics at Hella KGaA Hueck\&Co)," in International Research Conference in Dortmund 2013, 2013, pp. 4-7.

[28] SEI, "CMMI $®$ for Development, Version 1.3 - CMU/ SEI-2010-TR -033," 2010.

[29] PMI, A Guide to the Project Management Body of Knowledge ( $P M B O K^{\circledR}$ Guide ), Fifth Edit. Newton Square, Pennsylvania USA: Project Management Institute Inc, 2013.

[30] C. Pardo, F. García, F. Pino, M. Piattini Velthuis, and M. T. Baldassarre, "A reference ontology for harmonizing process-reference models," Revista Facultad de Ingeniería, Universidad de Antioquia N. 073, MedeIlin - Colombia, pp. 29-42, 2014.

[31] K. Beck and et al, "Manifiesto por el Desarrollo Ágil de Software," 2001.

[32] A. Vizcaíno, F. García, M. Piattini Velthuis, and S. Beecham, "A validated ontology for global software development," Comput. Stand. Interfaces, no. 46, pp. 66-78, 2016.

[33] Protégé, "The Protégé Ontology Editor and Knowledge Acquisition System," 2010. [Online]. Available: http://protege.stanford.edu. 
